УДК 532.785:66.065.5

\author{
ПЕРСПЕКТИВЫ ПРИМЕНЕНИЯ ТЕПЛОВЫХ НАСОСОВ \\ ПРИ ПРОВЕДЕНИИ КОМБИНИРОВАННЫХ \\ МАССООБМЕННЫХ ПРОЦЕССОВ
}

\author{
Г.А. Носов ${ }^{\circledR}$, М.В. Михайцов
}

Московский технологический университет (Институт тонких химических технологий имени М.В. Аомоносова), Москва 119571, Россия

@Aвтор для переписки, e-mail: nosovga@mail.ru

Рассмотрены перспективы использования тепловых насосов при разделении смесей путем сочетания различных массообменных проиессов, проведение которых связано с подводом и отводом тепловой энергии. При этом теплота, выделяющаяся на одних стадиях, может быть успешно использована на других стадиях разделения. При использовании тепловых насосов появляется возможность изменять температурные потенииалы тепловых потоков, ито в значительной мере повышает эффрективность рекуперативного теплообмена. Если при проведении массообменных проиессов образуется паровая фаза, то она может быть использована в качестве рабочего тела теплового насоса открытого типа. Если массообменные проиессы проводятся без образования паровых фраз, то следует применять тепловые насосы закрытого типа, в контуре которых ииркулируют различные промежуточные теплоносители. Кратко рассмотрены особенности разделения смесей путем сочетания проиессов фракиионной кристаллизаиии с выпариванием, растворением, фрракционным плавлением, дистилляиией и ректификацией с использованием компрессорных тепловых насосов открытого и закрытого типа. Для оиенки энергетической эфрфективности различных вариантов комбинированных проиессов и их сравнения с традииионным разделением предлагается использовать относительный расход условного топлива, что позволяет учитывать различную стоимость тепловой и электрической энергии, необходимой для проведения рассматриваемых проиессов. Произведено сопоставление между собой различных вариантов организаиии комбинированного разделения. При этом установлено, что их энергетическая эффрективность существенно зависит от состава исходной смеси, требований к продуктам разделения, а также от положения эвтектической точки на диаграммах "кидкость - твердая фраза". Показано, что использование тепловых насосов позволяет в несколько раз снизить энергетические затраты, а также расход хладагентов на проведение комбинированного разделения.

Ключевые слова: тепловые насосы, рекупераиия тепла, сопряженные проиессы, энергетические затраты, выпаривание, кристаллизаиия, растворение, фракиионное плавление, дистилляиия, ректификаиия.

\title{
PROSPECTS OF APPLYING HEAT PUMPS UPON CARRYING OUT COMBINED MASS TRANSFER PROCESSES
}

\section{G.A. Nosov ${ }^{\circledR}$, M.V. Mikhailov}

Moscow Technological University (M.V. Lomonosov Institute of Fine Chemical Technologies), Moscow 119571, Russia

@Corresponding author e-mail:nosovga@mail.ru

The prospects of using heat pumps for the separation of mixtures by combination of different mass transfer processes are considered, which is associated with the supply and removal of thermal energy. The heat released in some stages can be successfully used at other stages of separation. Using heat pumps makes it possible to change the temperature potentials of heat fluxes, which greatly improves the efficiency of regenerative heat transfer. If a vapor phase forms when carrying out mass transfer 
processes, it can be used as the working fluid of the heat pump of the open type. If mass transfer processes are carried out without the formation of vapor phases, it is necessary to apply heat pumps of the closed type, in the circuit of which a variety of intermediate heat transfer fluids circulate. The article briefly describes the features of mixtures separation by a combination of fractional crystallization by evaporation, dissolution, fractional melting, distillation and rectification with the use of compressor heat pumps of open and closed type. In order to assess the energy efficiency of different variants of combined processes and to compare them with traditional separation it is suggested to use the relative equivalent consumption, which makes it possible to take into account the different cost of thermal and electric energy required for carrying out the considered process of separation. Different variants of organizing the combined separation are compared. It was found that their energy efficiency significantly depends on the composition of the initial mixture, on requirements for the separation products, as well as on the position of the eutectic point on the liquid-solid phase charts. It was shown that the use of heat pumps allows reducing several times energy costs, as well as the consumption of refrigerants for combined separation.

Keywords: heat pumps, heat recovery, coupled processes, energy costs, evaporation, crystallization, dissolution, fractional melting, distillation, rectification.

Важной задачей химической технологии является снижение энергоемкости технологических процессов, которая весьма существенно отражается на себестоимости выпускаемой продукции. Учитывая это, во всех промышленно развитых странах в последнее десятилетие проводятся интенсивные исследования, направленные на снижение энергоемкости технологических процессов.

Одним из путей решения этой задачи является более рациональное использование низкопотенциальных источников тепловой энергии (теплоты, отводимой при охлаждении различных технологических потоков, теплоты конденсатов, вторичных паров, дистиллятов, отработанных сушильных агентов, растворителей, экстрагентов, сорбентов и др.). Частично эту задачу можно решить, организуя рекуперативный теплообмен между подводимыми, отводимыми и рециркулирующими потоками. Однако из-за низкой разности температур потоков эффективность рекуперативного теплообмена мала, а при отрицательной разности температур потоков рекуперативный теплообмен вообще становится невозможным. В результате этого теряется громадное количество тепла. При этом охлаждение или нагрев отводимых потоков часто требует значительных дополнительных затрат.

Проблему рационального использования низкопотенциальных тепловых источников в ряде случаев удается решить путем использования тепловых насосов, с помощью которых можно изменять в нужном направлении температурные потенциалы различных потоков [1-4]. По принципу действия тепловые насосы разделяют на парокомпрессионные, абсорбционные, адсорбционные, термохимические и термоэлектрические.

Наибольшее распространение в настоящее время получили парокомпрессионные тепловые насосы открытого и закрытого типа, работа которых основана на проведении процессов испаре- ния и конденсации рабочих тел (теплоносителей) при различных давлениях $[1,4,5]$. В тепловых насосах открытого типа в качестве «рабочих тел» (теплоносителей) выступают технологические потоки, находящиеся в парообразном состоянии; при сжатии таких потоков происходит повышение температуры их конденсации. В тепловых насосах закрытого типа в качестве «рабочих тел» используются какие-либо промежуточные теплоносители, которые циркулируют в замкнутом контуре между конденсатором и испарителем теплового насоса.

Для оценки энергетической эффективности применения компрессионные тепловых насосов часто используется коэффициент преобразования энергии $[1,5]$

$\varepsilon_{\mathrm{K}}=Q_{\mathrm{T}} / N_{\mathrm{K}}$,

где $Q_{\text {т }}$ - затраты тепловой энергии при осуществлении процесса без теплового насоса; $N_{\text {К }}$ - мощность компрессора теплового насоса.

Если величина $\varepsilon_{\mathrm{K}}$ больше единицы, применение теплового насоса оправдано. Однако здесь следует отметить, что $\varepsilon_{\mathrm{K}}>1$ является необходимым, но не достаточным условием целесообразности применения тепловых насосов. Это связано с тем, что при расчете коэффициента преобразования $\varepsilon_{\mathrm{K}}$ используются два вида энергии: тепловая и электрическая, затрачиваемая на привод компрессора. Эти виды энергии имеют разную стоимость. Как правило, стоимость электроэнергии обычно в 3-4 раза выше стоимости такого же количества тепловой энергии [1]. Поэтому мы считаем, что при оценке энергетической эффективности наряду с коэффициентом $\varepsilon_{\mathrm{K}}$ следует использовать расходы условного топлива на производство тепловой энергии в установках без теплового насоса $B_{\mathrm{T}}$ и расходы условного топлива при проведении процессов с применением тепловых на- 
сосов $B_{\mathrm{H}}$. Расходы условного топлива $B_{\mathrm{T}}$ и $B_{\mathrm{H}}$ составляют:

$B_{\mathrm{T}}=b_{\mathrm{T}} Q_{\mathrm{T}}$

$B_{\mathrm{H}}=b_{\ni} N_{\mathrm{K}}$

где $b_{\mathrm{T}}$ и $b_{\text {э }}$ - удельные расходы условного топлива на производство единицы тепловой и электрической энергии.

Если величина $B_{\mathrm{H}}$ меньше $B_{\mathrm{T}}$, то использование теплового насоса становится более выгодным по сравнению с обычным технологическим процессом. Для сравнения процессов разделения с использованием теплового насоса и без него можно также использовать относительный расход условного топлива [15]

$n_{\mathrm{T}}=B_{\mathrm{H}} / B_{\mathrm{T}}$

Если величина $n_{\mathrm{T}}<1$, то применение теплового насоса целесообразно.

В абсорбционных и адсорбционных тепловых насосах изменение температурных потенциалов потоков осуществляется за счет процессов растворения газообразных теплоносителей в жидких средах или же их сорбции на твердых сорбентах при различных давлениях [6-10]. К сорбционным тепловым насосам иногда относят установки, в которых происходит растворение или кристаллизация теплоносителей [6, 10]. В термохимических тепловых насосах перенос тепла с одного уровня на другой осуществляется в результате обратимых экзотермических и эндотермических реакций, протекающих при различных давлениях $[1,11]$.

В настоящее время наиболее широкое применение тепловые насосы нашли в теплоэнергетике [1-5]. Они также успешно используются при проведении ряда химико-технологических процессов, таких как выпаривание [1, 14-16], ректификация [1, 16-23], дистилляция [16, 24], сушка [25-27], растворение, кристаллизация [28-35], фракционное плавление [36, 37] и др.

Тепловые насосы могут также успешно использоваться при проведении различных комбинированных (гибридных) массообменных процессов [14, 38-40], что позволяет существенно расширить диапазоны возможного разделения. Особенно выгодно применять такие процессы для разделения смесей, на диаграммах равновесия которых имеются особые точки (азеотропные, эвтектические, перитектические и др.), а также области ограниченной растворимости, термического разложения и химического взаимодействия компонентов разделяемых смесей. Следует отметить, что практически все комбинированные про- цессы имеют значительное количество возможных вариантов их проведения [14, 39]. Рассмотрим более подробно особенности такого разделения.

Для выделения веществ из разбавленных растворов часто используются сочетания процессов выпаривания с однократной или многократной кристаллизацией $[41,42]$. При этом процесс выпаривания применяется для предварительного концентрирования исходных растворов или же рециркулирующих маточников с целью дополнительного выделения из них целевых компонентов. Особенно выгодно процесс выпаривания сочетать с выпарной кристаллизацией. Имеется несколько вариантов технологических схем проведения такого процесса с использованием одного или двух тепловых насосов. При применении одного теплового насоса вторичные пары выпарного аппарата и кристаллизатора объединяют. Далее, после сжатия компрессором теплового насоса, их направляют на обогрев выпарного аппарата или же кристаллизатора. Имеются схемы, в которых используются два компрессора для раздельного сжатия вторичных паров выпарного аппарата и кристаллизатора. Проведенные расчеты показали, что использование тепловых насосов для проведения такого разделения позволяет в $1.5-2$ раза снизить расход условного топлива [42].

Для очистки веществ от примесей довольно широко применяется процесс их перекристаллизации в среде различных растворителей. Перекристаллизация представляет собой многостадийный процесс. Она состоит из стадий растворения исходных веществ, одной или нескольких стадий кристаллизации, стадий промывки и регенерации растворителей $[13,40]$. Осуществление данного процесса обычно связано с довольно высокими затратами тепловой энергии.

Как известно [13, 14], при растворении большинства веществ имеет место поглощение теплоты, а при кристаллизации наблюдается выделение скрытой теплоты фазового превращения. Поэтому на стадиях растворения необходимо подводить определенное количество теплоты $Q_{\mathrm{H}}$, а на стадиях кристаллизации отводить количество теплоты $Q_{0}$. Величины тепловых потоков $Q_{\mathrm{H}}$ и $Q_{\mathrm{O}}$ часто соизмеримы и зависят от производительности установки, расхода растворителя, теплоты фазовых превращений, теплоемкости потоков, а также от выбранных параметров проведения стадий растворения и кристаллизации. Представляется целесообразным выделяющуюся на стадии кристаллизации теплоту использовать для нагрева растворов на стадии растворения. Однако при обычном процессе перекристаллизации это сделать не удается, так как температура на стадии растворения всегда выше температуры охлаждения на стадии кристаллизации. Для того, чтобы выделяющуюся теплоту на стадии кристаллизации $Q_{\text {о можно было }}$ 
использовать при проведении стадии растворения, необходимо осуществлять преобразование температурных потенциалов потоков $Q_{\mathrm{H}}$ и $Q_{\mathrm{O}}$. Это возможно при применении тепловых насосов $[43,44]$.

Одна из простейших схем однократной перекристаллизации с использованием теплового насоса представлена на рис. 1. В этой схеме для преобразования температурных потенциалов потоков используется тепловой насос закрытого типа, в контуре которого циркулирует промежуточный теплоноситель $G_{\mathrm{X}}$. Использовать в рассматриваемом процессе перекристаллизации тепловой насос открытого типа не представляется возможным, так как все материальные потоки находятся в конденсированном виде.
При проведении такого процесса исходное вещество

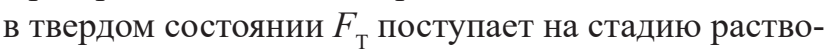
рения $\mathrm{H}$, куда подается растворитель $P$. В результате растворения получается раствор $F$ с концентрацией $x_{\mathrm{F}}$, который передается на стадию кристаллизации Кр. В результате охлаждения раствора на данной стадии до температуры $t_{\Phi}$ происходит процесс кристаллизации. При этом образуется суспензия, состоящая из кристаллической фазы $K$ и маточника $M$. Полученная на стадии кристаллизации суспензия далее направляется на стадию сепарации Ф, где производится отделение кристаллов от маточника. При этом получается очищенная кристаллическая фаза $S$ и маточник $L$, обогащенный примесями.

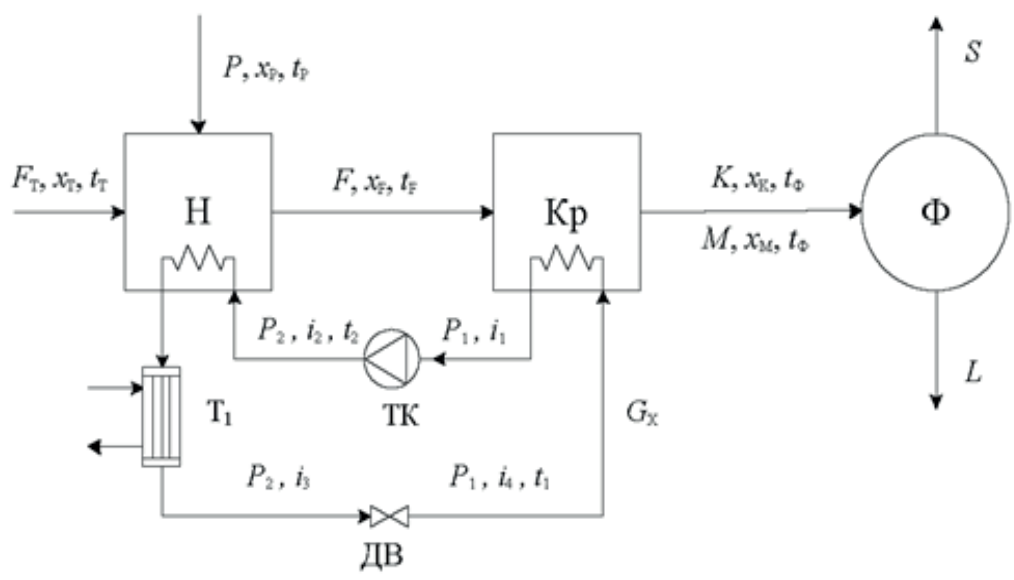

Рис. 1. Принципиальная схема однократной перекристаллизации с использованием теплового насоса с дополнительным охлаждением теплоносителя после стадии растворения

(обозначения здесь и далее см. в тексте).

Преобразование температурных потенциалов тепловых потоков осуществляется следующим образом: промежуточный теплоноситель $G_{\mathrm{X}}$ с энтальпией $i_{4}$ при температуре $t_{1}$ и давлении $p_{1}$ в парожидкостном состоянии поступает на стадию кристаллизации, где в результате теплообмена с кристаллизующимся раствором происходит его испарение. При этом раствор $F$ охлаждается до заданной температуры $t_{\Phi}$. Из кристаллизатора насыщенные пары теплоносителя подаются в компрессор ТК, где сжимаются от давления $p_{1}$ до давления $p_{2}$. При этом температура пара теплоносителя повышается от $t_{1}$ до $t_{2}$, а его теплосодержание изменяется от $i_{1}$ до $i_{2}$. После сжатия теплоноситель $G_{\mathrm{X}}$ поступает на стадию растворения Н, где в результате его охлаждения и полной конденсации выделяется теплота $Q_{\mathrm{H}}$, необходимая для проведения процесса растворения исходного вещества $F_{\mathrm{T}}$ в растворителе $P$. Сконденсированный теплоноситель проходит дроссельный вентиль ДВ. При этом его давление изменяется от $p_{2}$ до $p_{1}$. Полученная парожидкостная смесь снова направляется на стадию кристаллизации. Для компенсации возможной разности тепловых потоков $Q_{\mathrm{O}}$ и $Q_{\mathrm{H}}$ используется тепло- обменник $\mathrm{T}_{1}$. В результате теоретического анализа такого процесса разделения установлены технологические режимы, которые позволяют существенно (в 2-3 раза) снизить энергетические затраты на его осуществление. В работах $[43,44]$ был также рассмотрен процесс однократной перекристаллизации с регенерацией растворителя методом выпаривания. При этом на стадии регенерации используется тепловой насос открытого типа. В этом случае также достигается значительное понижение энергетических затрат.

Для разделения смесей на фракции, обогащенные теми или иными компонентами, довольно часто применяется процесс однократного или многостадийного фракционного плавления [13]. Для снижения энергетических затрат такого разделения также можно использовать тепловые насосы [35-37]. На рис. 2 показана одна из принципиальных схем однократного фракционного плавления. В данном случае исходная смесь $F$, состоящая из компонентов $A$ и $B$, в жидком состоянии при температуре $t_{\mathrm{F}}$ поступает на стадию кристаллизации Кр, где она охлаждается до температуры $t_{\text {}}$, ниже температуры ее полной кристаллизации. Затем образующуюся кристаллическую массу на ста- 
дии плавления ФП нагревают от температуры $t_{\text {к }}$ до температуры фракционирования $t_{\mathrm{H}}$, лежащей в диапазоне между температурами солидуса $t_{\mathrm{C}}$ и ликвидуса $t_{\text {л }}$ разделяемой смеси. При этом кристаллическая масса частично расплавляется. Полученная суспензия $K+M$ поступает на стадию сепарации $\Phi$, где про- исходит отделение кристаллической фазы $S$ с концентрацией $x_{\mathrm{S}}$ от маточника $L$, имеющего концентрацию $x_{\mathrm{L}}$. Маточник $L$ отбирается в качестве низкоплавкого продукта, обогащенного компонентом $B$, а кристаллическая фаза $S$ представляет собой конечный продукт, обогащенным высокоплавким компонентом $A$.

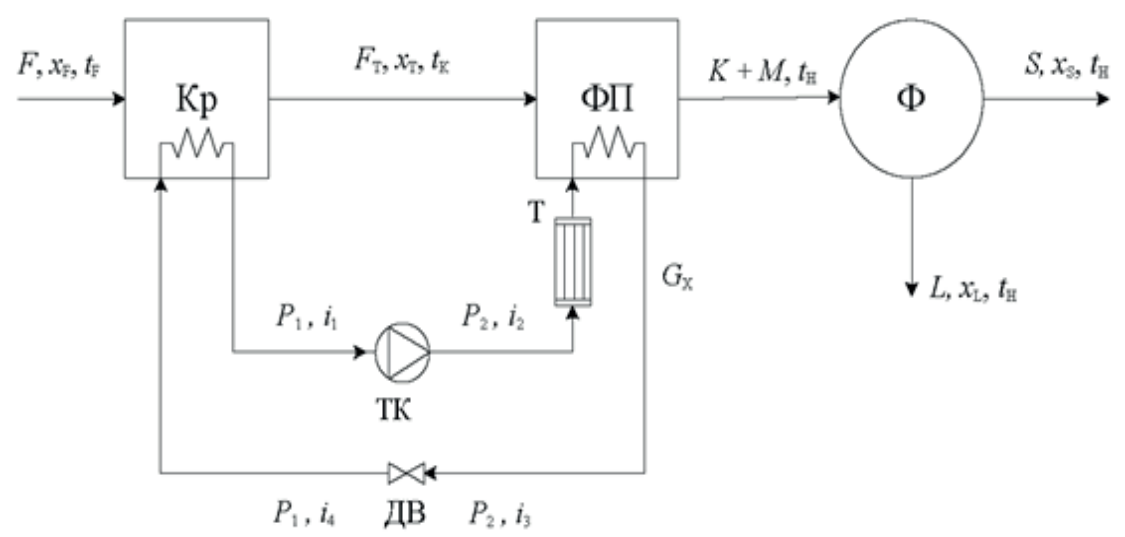

Рис. 2. Принципиальная схема однократного фракционного плавления.

Подвод и отвод тепла на стадиях плавления и кристаллизации может быть осуществлен с помощью теплового насоса закрытого типа, в контуре которого циркулирует промежуточный теплоноситель (рабочее тело) $G_{\mathrm{X}}$. В компрессоре ТК пары теплоносителя сжимаются от давления $p_{1}$ до давления $p_{2}$. При этом происходит повышение их теплосодержания (энтальпии) от $i_{1}$ до $i_{2}$. Нагретые таким образом пары теплоносителя $G_{\mathrm{x}}$ поступают в теплообменные элементы плавителя $Ф П$, где они в результате теплообмена с отвержденной смесью $F_{\text {T }}$ конденсируются. Далее промежуточный теплоноситель пропускается через дроссельный вентиль ДВ, в результате чего его давление снижается от $p_{2}$ до $p_{1}$. После дросселирования охлажденные влажные пары теплоносителя подаются в теплообменные элементы кристаллизатора, где происходит полное испарение теплоносителя. Таким образом, в рассматриваемом процессе плавитель выполняет функцию конденсатора теплового насоса, а кристаллизатор является испарителем теплового насоса. При проведении такого процесса разделения количество отводимого тепла на стадии кристаллизации $Q_{\mathrm{O}}$ может по величине не совпадать с количеством тепла, подводимым на стадию плавления $Q_{\mathrm{H}}$. В этом случае для компенсации разности тепловых потоков $Q_{\mathrm{O}}$ и $Q_{\mathrm{H}}$ необходимо предусмотреть теплообменник Т.

Имеются более сложные технологические схемы фракционного плавления с использованием тепловых насосов, в которых при охлаждении производится частичная кристаллизация исходной смеси $[13,36,37]$.

При разделении смесей путем сочетания процессов дистилляции и фракционной кристаллизации для снижения энергетических затрат можно использовать тепловые насосы как открытого, так и закрытого типа $[24,45]$. В этом случае также возможно несколько вариантов проведения разделения. На рис. 3 показана принципиальная схема одного из варианта разделения эвтектикообразующей смеси компонентов $A$ и $B$ с использованием теплового насоса закрытого типа. Исходная смесь $F$ с температурой $t_{\mathrm{F}}$ и концентрацией $x_{\mathrm{F}}$ поступает на стадию кристаллизации $\mathrm{Kp}_{\mathrm{B}}$, где охлаждается до температуры $t_{\text {Фв}}$. В результате образуется кристаллическая суспензия $K_{\mathrm{B}}+M_{\mathrm{B}}$, разделяемая на стадии сепарации $\mathrm{C}_{\mathrm{B}}$ на кристаллы $K_{\mathrm{B}}$ и маточник $M_{\mathrm{B}}$. Кристаллы $K_{\mathrm{B}}$, обогащенные компонентом $B$, отбираются в качестве одного из целевых продуктов, а маточник $M_{\text {в }}$ направляется на стадию дистилляции И, где нагревается до температуры $t_{\mathrm{u}}$. В результате частичного испарения маточника $M_{\mathrm{B}}$ образуется парожидкостная смесь $\Pi+W$, разделяемая на потоки кубового остатка $W$ и паров дистиллята П. Кубовый остаток $W$ возвращается на стадию кристаллизации Кр $\mathrm{B}_{\mathrm{B}}$, а дистиллят $\Pi$, обогащенный компонентом $A$, отбирается в качестве второго целевого продукта. Для утилизации тепла кубового остатка $W$ и дистиллята $\Pi$ используются теплообменники $\mathrm{T}_{1}$ и $\mathrm{T}_{2}$.

Частичное испарение рециркулирующего маточника $M_{\text {в }}$ на стадии дистилляции И осуществляется с использованием теплоты конденсации паров дистиллята П. Для этого пары дистиллята П пропускаются через теплообменник К, где они конденсируются, испаряя поток промежуточного теплоносителя $G_{\mathrm{X}}$, циркулирующего в замкнутом контуре теплового насоса. Пары теплоносителя $G_{\mathrm{X}}$ далее сжимаются компрессором теплового насоса ТК от давления $p_{1}$ до давления $p_{2}$. Сжатые пары $G_{\mathrm{X}}$ поступают в греющую камеру ис- 


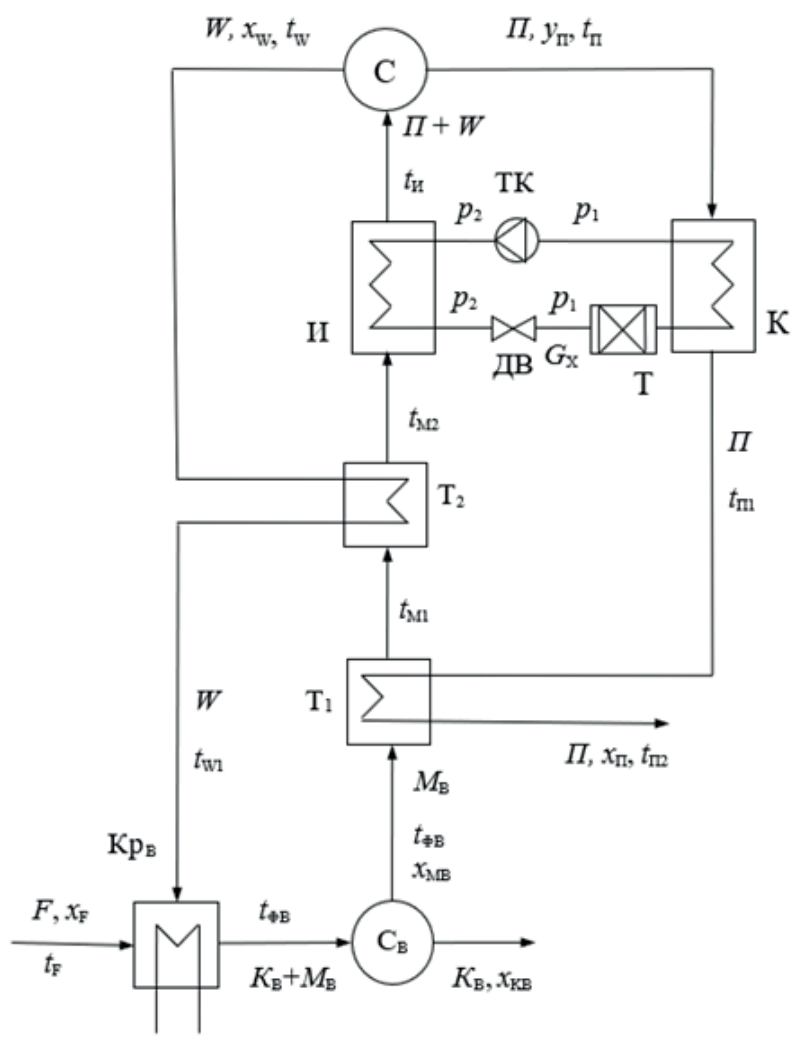

Рис. 3. Разделение путем сочетания дистилляции и одной стадии кристаллизации.

парителя И, где они конденсируются, нагревая поток маточника $M_{\text {в }}$ до температуры испарения $t_{\mathrm{u}}$. Сжатый конденсат теплоносителя $G_{\mathrm{x}}$ дросселируется от давления $p_{2}$ до давления $p_{1}$ и возвращается в теплообменник К. Для компенсации разности тепловых потоков $Q_{\text {и }}$ и $Q_{\mathrm{K}}$, подводимого в испаритель и отводимого в конденсаторе, в контуре циркуляции промежуточного теплоносителя предусмотрен теплообменник T.

Кроме описанного выше, возможны и другие варианты рассматриваемого сопряженного разделения с использованием тепловых насосов открытого и закрытого типа [24, 45]. Так, при концентрации исходной смеси $x_{\text {F }}$ выше эвтектической $x_{\mathrm{E}}$ исходная смесь $F$ может быть первоначально направлена на стадию кристаллизации Кр . При этом один из продуктов разделения получается в виде кристаллов компонента $A$, а другой продукт, обогащенный компонентом $B$, в виде кубового остатка $W$. Имеются варианты, в которых исходная смесь $F$ первоначально подается на стадию дистилляции с получением целевых продуктов разделения в виде потоков $K_{\text {в }}$ и $П$ или $K_{\mathrm{A}}$ и $W$. Анализ этих вариантов показал [24], что применение тепловых насосов позволяет в среднем в 1.5-2 раза снизить энергетические затраты на проведение такого разделения. Это достигается в результате того, что при применении тепловых насосов более эффективно используется теплота конденсации паров дистиллята для нагрева рециркулирующих маточников.
Особенно выгодно использовать тепловые насосы при разделении различных смесей сочетанием процессов ректификации и фракционной кристаллизации, так как ректификация является энергоемким процессом. В этом случае имеет место особенно большое количество возможных вариантов разделения [14, 39, 46-48].

На рис. 4 приведена принципиальная схема одного из вариантов процесса разделения бинарной эвтектической смеси путем сочетания ректификации с двумя стадиями кристаллизации. Исходная смесь $F$, состоящая из компонентов $A$ и $B$, с концентрацией $x_{\mathrm{F}}$ больше эвтектической $x_{\mathrm{E}}$, первоначально подается на стадию кристаллизации компонента $A$, где охлаждается до температуры $t_{\text {ФА }}$. После разделения полученной суспензии кристаллическая фаза $K_{\mathrm{A}}$, представляющая практически чистый компонент $A$, отбирается в качестве одного из продуктов разделения, а маточник $M_{\mathrm{A}}$ вместе с маточником $M_{\mathrm{B}}$ со стадии кристаллизации компонента $B$ подается на стадию ректификации. При ректификации маточников $M_{\mathrm{A}}$ и $M_{\mathrm{B}}$ получается кубовый остаток $W$, обогащенный компонентом $B$, и дистиллят $П$, обогащенный компонентом $A$. Последний возвращается на стадию кристаллизации компонента $A$. В свою очередь, кубовый остаток $W$ подается на стадию кристаллизации компонента $B$, где он охлаждается до температуры $t_{\text {Фв}}$. При этом образуется суспензия $K_{\mathrm{B}}+M_{\mathrm{B}}$, после разделения которой получается продукт $K_{\mathrm{B}}$, представляющий собой практически чистый компонент $B$.

В данном варианте пары $D$, выходящие из ректификационной колонны РК, подаются в компрессор теплового насоса ТК, где они сжимаются от давления $p_{1}$ до $p_{2}$. Далее сжатые пары направляются в конденсатор К, где они конденсируются, нагревая тем самым объединенный маточник $M_{\Sigma}$ до температуры $t_{\mathrm{M} 3}$. Образующийся конденсат проходит через дроссельный вентиль ДВ, в результате чего его давление снижается от $p_{2}$ до $p_{1}$. Затем конденсат разделяется на дистиллят П и флегмовый поток $L$. Поток $L$ возвращается в ректификационную колонну РК в качестве флегмы, а дистиллят П после его охлаждения в теплообменнике $\mathrm{T}_{1}$ от температуры $t_{\Pi}$ до $t_{\Pi 1}$ за счет теплообмена с объединенным маточником $M_{\Sigma}$, поступающим со стадии кристаллизации $\mathrm{Kp}_{\mathrm{A}}$ и К $\mathrm{p}_{\mathrm{B}}$, подается на стадию кристаллизации К $\mathrm{p}_{\mathrm{A}}$. При этом маточник $M_{\Sigma}$ нагревается от температуры $t_{\mathrm{M}}$ до $t_{\mathrm{M} 1}$. Отводимый из колонны кубовый остаток $W$ направляется в теплообменник $\mathrm{T}_{2}$, где охлаждается от температуры $t_{\mathrm{w}}$ до $t_{\mathrm{W} 1}$ в результате теплообмена с потоком маточника $M_{\Sigma}$, который нагревается от $t_{\mathrm{M} 1}$ до $t_{\mathrm{M} 2}$.

В описанном выше варианте ректификация используется в основном для перехода через эвтектическую точку $E$. Если концентрация исходной смеси $x_{\mathrm{F}}<x_{\mathrm{E}}$, то ее можно подать или на стадию кристаллизации ком- 


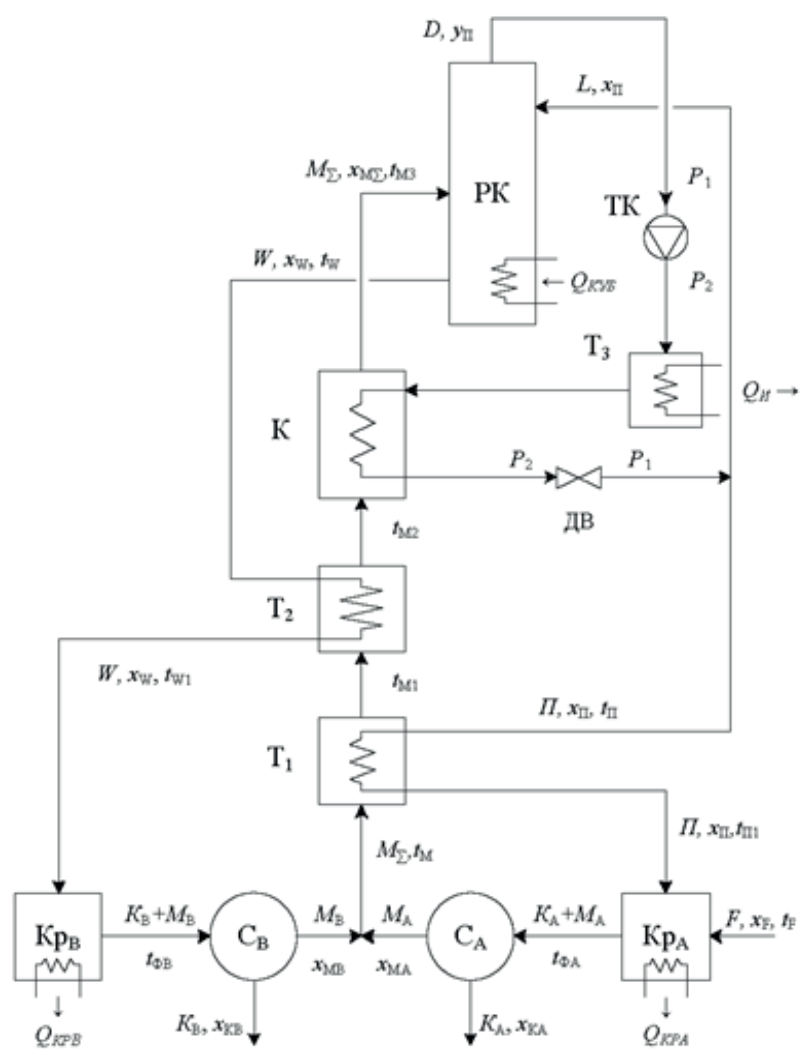

Рис. 4. Принципиальная схема разделения путем сочетания ректификации с двумя стадиями

кристаллизации с использованием теплового насоса открытого типа.

понента $B$, или же на стадию ректификации. Имеются варианты, в которых ректификация сочетается с одной стадией кристаллизации. При этом один из продуктов разделения получается в виде кристаллов компонента $A$ или $B$, а другой продукт отбирается со стадии ректификации в виде дистиллята П или же кубового остатка $W$.
Для оценки эффективности использования тепловых насосов при сочетании процессов ректификации и кристаллизации нами проведены расчеты процесса разделения ряда эвтектикообразующих бинарных смесей (вода - пропионовая кислота, бензол $-n$-ксилол, нафталин - дифенил и др.) с использованием различных вариантов. Для одного из вариантов разделения в таблице приведены тепловые затраты, расход электроэнергии $N_{\text {к }}$ и расход условного топлива для трех режимов: разделение без рекуперативного теплообмена и без использования теплового насоса, разделение с использованием обычного рекуперативного теплообмена между потоками и разделение с использованием теплового насоса. Из представленных данных видно, что организация обычного рекуперативного теплообмена позволяет снизить общие тепловые затраты (а, следовательно, и расход условного топлива) примерно на 15-20\%. При применении же теплового насоса расход условного топлива сокращается в 3-4 раза по сравнению с разделением без теплового насоса.

Выгодно также использовать тепловые насосы при разделении и очистке веществ от примесей путем сочетания процессов сублимации и десублимации. Как известно [49], эти процессы имеют наиболее высокие значения теплот фазовых превращений. Тепловые насосы можно также применять при разделении газообразных смесей абсорбцией или же адсорбцией с последующей регенерацией жидких и твердых сорбентов методом десорбции. При сорбции, как известно [50], наблюдается выделение тепла, а для проведения десорбции обычно требуется подвод тепловой энергии. Наконец, тепловые насосы можно эффективно использовать при проведении многих реакционных процессов в сочетании с различными массообменными процессами.

Таблица. Показатели режимов разделения для варианта с использованием одной стадии кристаллизации

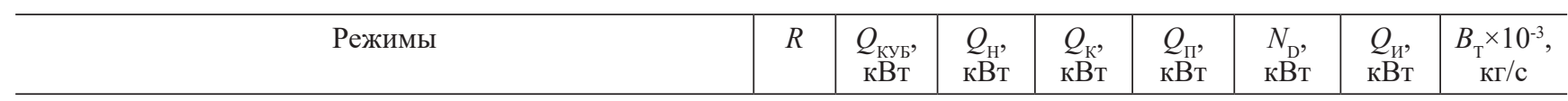

\begin{tabular}{l|c|c|c|c|c|c|c|c|c}
\hline \multicolumn{1}{c}{ Вода - пропионовая кислота (F $=1$ кг/с, $x_{\mathrm{F}}=50 \%$ масс., $y_{\Pi}=92 \%$ мол., $x_{\mathrm{W}}=10 \%$ мол.) } \\
\hline $\begin{array}{l}\text { Процесс без рекуперации тепла и без использования } \\
\text { теплового насоса }\end{array}$ & 0.7 & 381.5 & 209.4 & 360.3 & - & - & - & 24.7 \\
\hline $\begin{array}{l}\text { Процесс с рекуперацией тепла и без использования } \\
\text { теплового насоса }\end{array}$ & 0.7 & 381.5 & 34.0 & 360.3 & - & - & - & 17.4 \\
\hline
\end{tabular}
теплового насоса

Процесс с рекуперащией тепла и с использованием теплового насоса

\begin{tabular}{l|c|c|c|c|c|c|c|c}
\hline $\begin{array}{l}\text { Подача потока маточника } \\
\text { в колонну при температуре кипения }\end{array}$ & 0.7 & 381.5 & - & 360.3 & 388.4 & 43.9 & 354.4 & 5.4 \\
\hline $\begin{array}{l}\text { Подача потока маточника } \\
\text { в колонну с долей пара } \psi \text { ч } 0.6\end{array}$ & 1.4 & 159.1 & - & 492.2 & 530.5 & 60.0 & 142.0 & 6.8 \\
\hline $\begin{array}{l}\text { Подача потока маточника } \\
\text { в колонну в виде насыщенного пара }\end{array}$ & 2.4 & 159.9 & - & 703.0 & 757.7 & 85.8 & 159.3 & 8.4 \\
\hline
\end{tabular}

Бензол $-n$-ксилол ( $\mathrm{F}=1$ кг/c, $x_{\mathrm{F}}=70 \%$ масс., $y_{\Pi}=98 \%$ масс., $x_{\mathrm{W}}=10 \%$ мол.)

Процесс без рекуперации тепла и без использования теплового насоса

\begin{tabular}{|l|l|l|l|l|l|l|l}
0.3 & 217.9 & 138.9 & 206.6 & - & - & - & 14.9 \\
\hline
\end{tabular}


Таблица. Окончание

\begin{tabular}{|c|c|c|c|c|c|c|c|c|}
\hline $\begin{array}{l}\text { Процесс с рекуперацией тепла и без использования } \\
\text { теплового насоса }\end{array}$ & 0.3 & 217.9 & 34.4 & 206.6 & - & - & - & 10.5 \\
\hline \multicolumn{9}{|c|}{ Прочесс с рекуперацией тепла и с использованием теплового насоса } \\
\hline $\begin{array}{l}\text { Подача потока маточника } \\
\text { в колонну при температуре кипения }\end{array}$ & 0.3 & 217.9 & - & 206.6 & 206.6 & 31.5 & 172.2 & 4.4 \\
\hline $\begin{array}{l}\text { Подача потока маточника } \\
\text { в колонну с долей пара } \psi=0.7\end{array}$ & 0.9 & 112.8 & - & 295.9 & 327.8 & 49.9 & 99.1 & 5.4 \\
\hline $\begin{array}{l}\text { Подача потока маточника } \\
\text { в колонну в виде насыщенного пара }\end{array}$ & 1.3 & 104.1 & - & 360.9 & 399.8 & 60.9 & 97.3 & 6.2 \\
\hline
\end{tabular}

\section{Список литературы:}

1. Янтовский Е.И., Левин Л.А. Промышленные тепловые насосы. М.: Энергоиздат, 1989. 128 с.

2. Горшков В.Г. Тепловые насосы. Аналитический обзор // Справочник промышленного оборудования. 2004. № 2. С. 47-80.

3. Энергетическое оборудование для использования нетрадиционных и возобновляемых источников энергии / Под ред. В.И. Виссарионова. М.: ООО фирма «ВИЭН», 2004. 448 с.

4. Амерханов Р.А. Тепловые насосы. М.: Энергоатомиздат, 2005. 160 с.

5. Бараненко А.В., Бухарин Н.Н., Пекарев В.И., Тимофеевский Л.С. Холодильные машины / Под общ. ред. Л.С. Тимофеевского. СПб.: Политехника, 2006. 944 c.

6. Орехов И.И., Тимофеевский Л.С., Караван С.К. Абсорбционные преобразователи теплоты. Л.: Химия, 1989. 208 с.

7. Ring T.A., Dirksen J.A. Absorption heat pumps having improved efficiency using a crystallizationinhibiting additive. University of Utah, 2008. 136 p.

8. Winnington T.L., Grin R.J., Lorton R., Uselton R.B. Absorption heat pump and method of his work. Interoteks Limited Publ., 2001. 126 p.

9. Бараненко А.В., Тимофеевский Л.С., Попов А.В. Абсорбционные преобразователи теплоты. СПб.: СПб-ГУН и ПТ, 2005. 338 с.

10. Пивинский А.А. Оценка эффективности парокомпрессионных тепловых насосов и абсорбционных бромистолитиевых понижающих термотрансформаторов: дис. ... канд. техн. наук. СПб., 2005. 209 с.

11. Kudra T. Heat-pump drying // Advanced Drying Technologies. 2nd ed. New York, 2008. 500 p.

12. Култыгин Я.Б., Митрясов П.В., Новиков А.Г., Плетнев А.В. Использование нетрадиционных возобновляемых источников энергии. Л.: ЦНИИ «Румб», 1989. 114 с.

13. Гельперин Н.И., Носов Г.А. Основы техники фракционной кристаллизации. М.: Химия, 1986. 304 с.

14. Айнштейн В.Г., Захаров М.К., Носов Г.А., Захаренко В.В., Зиновкина Т.В., Таран А.Л., Костанян A.Е. [и др]. Общий курс процессов и аппаратов хи-

\section{References:}

1. Yantovskii E. I., Levin L.A. Industrial heat pumps. M.: Energoizdat Publ., 1989. 128 p. (in Russ.).

2. Gorshkov V.G. Heat pumps. Analytical overview // Spravochnik promyshlennogo oborudovaniya (Catalog of Industrial Equipment). 2004. № 2. P. 47-80. (in Russ.).

3. Energy equipment for the use of alternative and renewable energy sources / Ed. by V.I. Vissarionov. Moscow: «VIEN» Publ., 2004. 448 p. (in Russ.).

4. Amerkhanov R.A. Heat pumps. Moscow: Energoatomizdat Publ., 2005. 160 p. (in Russ.).

5. Baranenko A.V., Bukharin N. N., Pekarev V.I., Timofeevskiy S.L. Refrigerating apparatus / Under the general editorship of S.L. Timofeevskiy. SaintPetersburg: Politekhnika Publ., 2006. 944 p. (in Russ.).

6. Orekhov I.I., Timofeevsky L.S., Karavan S.K. Absorption heat converters. Leningrad: Khimiya Publ., 1989. 208 p. (in Russ.).

7. Ring T.A., Dirksen J.A. Absorption heat pumps having improved efficiency using a crystallizationinhibiting additive. University of Utah, 2008. 136 p.

8. Winnington T.L., Grin R.J., Lorton R., Uselton R.B. Absorption heat pump and method of his work. Interoteks Limited Publ., 2001. 126 p.

9. Baranenko A.V., Timofeevsky L.S., Popov A.V. Absorption of heat converters. Saint-Petersburg: SPbGUN and PT Publ., 2005. 338 p. (in Russ.).

10. Pivinskii A.A. Evaluation of the efficiency of vapor compression heat pumps and absorption thermotransformers lithium bromide decreasing: diss. ... Ph.D. (Eng.) Saint-Petersburg, 2005. 209 p. (in Russ.).

11. Kudra T. Heat-pump drying // Advanced Drying Technologies. 2nd ed. New York, 2008. 500 p.

12. Kultygin Ya.B., Mitresov P.V., Novikov A.G., Pletnev A.V. The use of non-renewable energy sources. Leningrad: TsNII "Rumb", 1989. 114 p. (in Russ.).

13. Gelperin N.I., Nosov G.A. Fundamentals of fractional crystallization techniques. Moscow: Khimiya Publ., 1986. 304 p. (in Russ.).

14. Ainshtein V.G., Zakharov M.K., Nosov G.A., Zakharenko V.V., Zinovkina T.V., Taran A.L., Kostaniyan A.E. [et al.] Processes and devices of chemical technology. M: Terevinf Publ., 2015. 1784 p. (in Russ.). 
мической технологии. Общий курс в 2 кн. М.: Теревинф, 2015. 1784 с.

15. Лыскова М.В., Кузнецова И.К., Дмитриев Е.А. Техноэкономический анализ выпаривания с тепловым насосом // Успехи в химии и хим. технол. 2006. № 2. C. 77-80.

16. Захаров М.К., Носов Г.А., Мясоеденков В.Н. Применение тепловых насосов в процессах химической технологии // Ученые записки МИТХТ. 2002. Вып. 6. С. 54-63.

17. Лебедев Ю.Н., Александров И.А., Зайцева T.M. Ректификация смесей близкокипящих компонентов с использованием тепловых насосов // Химия и технол. топлив и масел. 2010. № 4. С. 10-13.

18. Ghazal K., Majid A., Heidari S. H., Reza S. G. Distillation column controllability analysis through heat pump integration // Chem. Eng. \& Process. 2015. V. 97. P. 23-37.

19. Yuliang L., Jian Z., Lumin L., Lanyi S., Cheng Z. Heat pump assisted reactive and azeotropic distillations in dividing wall columns // Chem. Eng. \& Process. 2015. V. 95. P. 289-301.

20. Van Duc L.N., Moonyong L. A hybrid technology combining heat pump and thermally coupled distillation sequence for retrofit and debottlenecking // Energy. 2015. V. 81. P. 103-110

21. Yus D.C., Le Quang M., Mohd Shariq K., KeeKahb K., Alireza B., Moonyong L. Optimal design of advanced distillation configuration for enhanced energy efficiency of waste solvent recovery process in semiconductor industry // Energy Conversion and Management. 2015. V. 102. P. 92-103.

22. Хоменков А.С., Ильина С.И. Энергосбережение в процессах ректификации с применением тепловых насосов // Успехи в химии и хим. технол. 2017. Т. 31. № 5 (186). С. 17-19.

23. Разумовский Г.Н., Селиваненко И.Л. Применение компрессионного теплового насоса для рекуперации энергии в процессе ректификации воды под вакуумом // Хим. пром. сегодня. 2017. № 2. С. 17-26.

24. Жильцов В.С. Разделение эвтектикообразующих смесей сочетанием дистилляции и фракционной кристаллизации с использованием тепловых насосов: дис. ... канд. техн. наук. М.: МИТХТ, 2017. 200 с.

25. Гузев О.Ю., Гончарова С.В. Моделирование кинетики многостадийной сушки биоматериала в сушилке псевдоожиженного слоя с тепловым насосом // Успехи в химии и хим. технол. 2006. № 1. С. 78-82.

26. Hawlader M.N.A., Perera C.O., Tian M. Properties of modified atmosphere heat pump dried foods // J. Food Eng. 2006. V. 74. № 3. P. 392-401.

27. Захаров М.К. Об эффективности применения тепловых насосов в процессах сушки влажных материалов // Хим. пром. 2002. № 9. С. 43-47.

28. Носов Г.А., Кесоян Г.А., Мясоеденков В.М.,
15. Lyskova M.V., Kuznetsova I.K., Dmitriev E.A. Techno-economic analysis of the evaporation with a heat pump // Uspekhi v himii i himicheskoj tekhnologii (Advances in Chemistry and Chemical Technology). 2006. № 2. P. 77-80. (in Russ.).

16. Zakharov M.K., Nosov G.A., Myasoedov V.N. The use of heat pumps in chemical technology processes // Uchenye zapiski MITHT (Scientific Notes of MITHT). 2002. V. 6. P. 54-63. (in Russ.).

17. Lebedev Yu.N., Aleksandrov I.A., Zaitseva T.M. Distillation of a mixture of close components with the use of heat pumps // Khimiya i tekhnologiya topliva i masel (Chemistry and Technology of Fuels and Oils). 2010 № 4. P. 10-13. (in Russ.).

18. Ghazal K., Majid A., Heidari S. H., Reza S. G. Distillation column controllability analysis through heat pump integration // Chem. Eng. \& Process. 2015. V. 97. P. 23-37.

19. Yuliang L., Jian Z., Lumin L., Lanyi S., Cheng Z. Heat pump assisted reactive and azeotropic distillations in dividing wall columns // Chem. Eng. \& Process. 2015. V. 95. P. 289-301.

20. Van Duc L.N., Moonyong L. A hybrid technology combining heat pump and thermally coupled distillation sequence for retrofit and debottlenecking // Energy. 2015. V. 81. P. 103-110

21. Yus D.C., Le Quang M., Mohd Shariq K., KeeKahb K., Alireza B., Moonyong L. Optimal design of advanced distillation configuration for enhanced energy efficiency of waste solvent recovery process in semiconductor industry // Energy Conversion and Management. 2015. V. 102. P. 92-103.

22. Khomenkov A.S., Ilyin S.I. Energy saving in the processes of rectification with the use of heat pumps // Uspekhi v himii i himicheskoj tekhnologii (Advances in Chemistry and Chemical Technology). 2017. V. 31. № 5 (186). P. 17-19. (in Russ.).

23. Razumovsky G.N., Selivanenko I.L. The use of a compression heat pump for energy recovery in the process of distillation of water under vacuum // Khimicheskaya promyshlennost' segodnya (Chemical Industry Today). 2017 № 2. P. 17-26. (in Russ.).

24. Zhil'tsov V.S. Separation of eutectic mixtures by a combination of single distillation and fractional crystallization processes using heat pumps: diss. ... Ph.D. (Eng.). Moscow, 2017. 200 p. (in Russ).

25. Guzev O.Yu., Goncharov S.V. Modeling of the kinetics of multistage drying of biological material in the dryer fluidized bed with a heat pump // Uspekhi v himii i himicheskoj tekhnologii (Advances in Chemistry and Chemical Technology). 2006. № 1. P. 78-82. (in Russ.).

26. Hawlader M.N.A., Perera C.O., Tian M. Properties of modified atmosphere heat pump dried foods // J. Food Eng. 2006. V. 74. № 3. P. 392-401.

27. Zakharov M.K. About the effectiveness of 
Наби Лай Бангура. Выпарная кристаллизация с использованием теплового насоса // Хим. технология. 2002. № 5. C. 32-35.

29. Носов Г.А., Мясоеденков В.М., Кхафи Али, Наби Лай Бангура. Адиабатная кристаллизация с использованием теплового насоса // Хим. технология. 2004. № 3. C. 35-40.

30. Al-Harahsheh Adnan M. Theoretical analyses of energy saving in a direct contact evaporative crystallization through the installation of heat pump // Desalination. 2010. V. 251. № 1-3. P. 47-52.

31. Al-Harahsheh Adnan M. A heat pump in a countercurrent crystallization process // Appl. Therm. Eng. 2005. V. 25. P. 545-555.

32. Al-Harahsheh Adnan M. Theoretical analyses of energy saving in indirect contact evaporative crystallization by using combined cycle of vapor recompression heat pump and throttling valve // Jordan J. Mechan. \& Ind. Eng. 2010. V. 4. № 3. P. 358-363.

33. Мясоеденков В.М., Носов Г.А., Хайбулина Е.М. Уваров М.Е. Противоточная кристаллизация с тепловым насосом // Вестник МИТХТ. 2007. Т. 2. № 6. С. $75-83$.

34. Носов Г.А., Уваров М.Е., Мясоеденков В.М. Перекристаллизация веществ из растворов с применением теплового насоса // Вестник МИТХТ. 2009. T. 4. № 1. С. 64-68.

35. Мясоеденков В.М., Хайбулина Е.М. Эффективность использования теплового насоса в установке противоточной кристаллизации с питанием в центр // Успехи в химии и хим. технол. 2010. Т. 24. № 11. С. $106-110$.

36. Мясоеденков В.М., Носов Г.А., Разина М.Н. Фракционное плавление с использованием теплового насоса // Вестник МИТХТ. 2007. Т. 2. № 1. С. 18-25.

37. Хайбулина Е.М. Разделение смесей методами фракционного плавления и противоточной фракционной кристаллизации с использованием тепловых насосов: дис. ... канд. техн. наук. М.: МИТХТ, 2013. $207 \mathrm{c}$

38. Clasen H. Optimale Kombination von Kristallisation und Rektifikation zur Trennung nicht - isomerierbarer Isomerenqemischen // Chemie Ing. Techn. 1967. В. 39. Heft 22. S. 1279-1285.

39. Гельперин Н.И., Носов Г.А. Разделение смесей путем сочетания некоторых массообменных процессов // Хим. пром. 1979. №11. С. 677-681.

40. Горштейн Г.И. Циклы многократной кристаллизации // Труды ИРЕА. 1951. Вып. 20. С. 96-109.

41. Носов Г.А., Сорокина В.И., Терехова Ю.О. Двухкорпусная выпарная кристаллизация с использованием тепловых насосов // Хим. технология. 2013. № 9. С. 570-575.

42. Бельская В.И. Разработка энергосберегаю- heat pumps in drying processes of wet materials // Khimicheskaya tekhnologiya (Chemical Industry). 2002. № 9. P. 43-47. (in Russ.).

28. Nosov G.A., Kesoyan G.A., Myasoedenkov V.M., Nabi Lai Bangura. Evaporation-crystallization with the use of heat pump // Khimicheskaya tekhnologiya (Chemical Technology). 2002. № 5. P. 32-35. (in Russ.).

29. Nosov G.A., Myasoedenkov V.M., Khafi Ali, Nabi Lai Bangura. Adiabatic crystallization using heat pump // Khimicheskaya tekhnologiya (Chemical Technology). 2004. № 3. P. 35-40. (in Russ.).

30. Al-Harahsheh Adnan M. Theoretical analyses of energy saving in a direct contact evaporative crystallization through the installation of heat pump // Desalination. 2010. V. 251. № 1-3. P. 47-52.

31. Al-Harahsheh Adnan M. A heat pump in a countercurrent crystallization process // Appl. Therm. Eng. 2005. V. 25. P. 545-555.

32. Al-Harahsheh Adnan M. Theoretical analyses of energy saving in indirect contact evaporative crystallization by using combined cycle of vapor recompression heat pump and throttling valve // Jordan J. Mechan. \& Ind. Eng. 2010. V. 4. № 3. P. 358-363.

33. Myasoedenkov V.M., Nosov G.A., Khaybulina E.M., Uvarov M.E. Countercurrent crystallization with heat pump // Vestnik MITHT (Fine Chemical Technologies). 2007. V. 2. № 6. P. 75-83. (in Russ.).

34. Nosov G.A., Uvarov M.E., Myasoedenkov V.M. Recrystallization of substances from solutions by the use of a heat pump // Vestnik MITHT (Fine Chemical Technologies). 2009. V. 4. № 1. P. 64-68. (in Russ.).

35. Myasoedenkov V.M., Khaybulina E.M. Efficiency of the heat pump in the installation of countercurrent crystallization with food in the center // Uspekhi v himii i himicheskoj tekhnologii (Advances in Chemistry and Chemical Technology). 2010. V. 24. № 11. P. 106-110. (in Russ.).

36. Myasoedenkov V.M., Nosov G.A. Razina M.N. Fractional melting using the heat pump // Vestnik MITHT (Fine Chemical Technologies). 2007. V. 2. № 1. P. 18-25. (in Russ.).

37. Khaybulina E.M. Separation of mixtures by fractional melting and countercurrent fractional crystallization with the use of heat pumps: diss. ... Ph.D. (Eng.). Moscow, 2013. 207 p. (in Russ).

38. Clasen H. Optimale Kombination von Kristallisation und Rektifikation zur Trennung nicht - isomerierbarer Isomerenqemischen // Chemie Ing. Techn. 1967. B. 39. Heft 22. S.1279-1285.

39. Gel'perin N.I., Nosov G.A. Separation of mixtures by combining some mass-exchange processes // Khimicheskaya promyshlennost' (Chemical Industry). 1979. № 11. P. 677-681. (in Russ.).

40. Gorshtein G.I. Repeated cycles of crystallization // Trudy IREA (Proceedings of IREA). 
щих вариантов выпарной кристаллизации: дис. ... канд. техн. наук. М.: МИТХТ, 2013. 162 с.

43. Уваров М.Е. Перекристаллизация веществ из растворов с использованием тепловых насосов: дис. ... канд. техн. наук. М.: МИТХТ, 2013. 171 с.

44. Носов Г.А., Уваров М.Е. Двухстадийная перекристаллизация с регенерацией растворителя // Тонкие химические технологии. 2017. Т. 12. № 1. С. $50-56$.

45. Носов Г.А., Бельская В.И., Жильцов В.С. Разделение смесей путем сочетания процессов кристаллизации и непрерывной дистилляции с использованием тепловых насосов // Вестник МИТХТ. 2014. T. 9. № 3. С. 31-35.

46. Карасев В.В. Разделение бинарных смесей методом сочетания процессов кристаллизации и ректификации: дис. ... канд. техн. наук. М.: МИТХТ, 1977. $200 \mathrm{c.}$

47. Баншац Р.Ш. Разделение бинарных неидеальных смесей путем сочетания процессов ректификации и фракционной кристаллизации: дис. ... канд. техн. наук. М.: МИТХТ, 1985. 192 с.

48. Носов Г.А., Михайлов М.В., Абсаттаров А.И. Разделение смесей путем сочетания процессов ректификации и фракционной кристаллизации // Тонкие химические технологии. 2017. Т. 12. № 3. С. 44-51.

49. Горелик А.Г. Десублимация в химической промышленности. М.: Химия, 1986. 272 с.

50. Рамм В.М. Абсорбция газов. М.: Химия, 1976. $656 \mathrm{c}$.
1951. V. 20. P. 96-109. (in Russ.).

41. Nosov G.A., Sorokin V.I., Terekhova Yu.O. Two section evaporation-crystallization with the use of heat pumps // Khimicheskaya tekhnologiya (Chemical Technology). 2013. № 9. P. 570-575. (in Russ.).

42. Bel'skaya V.I. Development of energy saving options evaporation-crystallization: diss. ... Ph.D. (Eng.). Moscow, 2013. 162 p. (in Russ).

43. Uvarov M. E. Recrystallization of substances from solutions with the use of heat pumps: diss. ... Ph.D. (Eng.). Moscow, 2013. 171 p. (in Russ.).

44. Nosov G.A., Uvarov M.E. Two stage recrystallizations with solvent recovery // Tonkiye khimicheskiye tekhnologii (Fine Chemical Technologies). 2017. V. 12. № 1. P. 50-56. (in Russ.).

45. Nosov G.A., Bel'skaya V.I., Zhil'tsov V.S. Separation of mixtures by combination of crystallization and continuous distillation with the use of heat pumps // Vestnik MITHT (Fine Chemical Technologies). 2014. V. 9. № 3. P. 31-35. (in Russ.).

46. Karasev V.V. Separation of binary mixtures by a combination of crystallization and distillation: diss. ... Ph.D. (Eng.). Moscow, 1977. 200 p. (in Russ.).

47. Banshats R.Sh. Separation of nonideal binary mixtures by combining rectification processes and fractional crystallization: diss. ... Ph.D. (Eng.). Moscow, 1985. 192 p. (in Russ.).

48. Nosov G.A., Mikhailov M.V., Absattarov A.I. Separation of mixtures by combining rectification and fractional crystallization processes // Tonkiye khimicheskiye tekhnologii (Fine Chemical Technologies). 2017. V. 12. № 3. P. 44-51. (in Russ.).

49. Gorelik A.G. Desublimate in the chemical industry. Moscow: Khimiya Publ., 1986. 272 p. (in Russ.).

50. Ramm V.M. Absorption of gases. Moscow: Khimiya Publ., 1976. 656 p. (in Russ.).

\section{Об авторах:}

Носов Геннадий Алексеевич, доктор технических наук, профессор кафедры процессов и аппаратов химической технологии имени Н.И. Гельперина Института тонких химических технологий имени М.В. Ломоносова ФГБОУ ВО «Московский технологический университет» (Россия, 119571, Москва, пр-т Вернадского, д. 86)

Muxaйлов Muxaur Bладuмupoвич, аспирант кафедры процессов и аппаратов химической технологии имени Н.И. Гельперина Института тонких химических технологий имени М.В. Ломоносова ФГБОУ ВО «Московский технологический университет» (Россия, 119571, Москва, пр-т Вернадского, д. 86).

\section{About the authors:}

Gennady A. Nosov, Dr.Sc. (Engineering), Professor of the N.I. Gel'perin Chair of Processes and Apparatus of Chemical Technologies, M.V. Lomonosov Institute of Fine Chemical Technologies, Moscow Technological University (86, Vernadskogo Pr., Moscow, 119571, Russia).

Mikhail V. Mikhailov, Post-Graduate Student of the N.I. Gel'perin Chair of Processes and Apparatus of Chemical Technologies, M.V. Lomonosov Institute of Fine Chemical Technologies, Moscow Technological University (86, Vernadskogo Pr., Moscow, 119571, Russia). 